\title{
THE OCCURRENCE OF LOOSE SMUT RESISTANCE IN 42, 28 AND 14, CHROMOSOME WHEATS ${ }^{1}$
}

\author{
R. G. ANDERSON \\ Canada Department of Agriculture, Winnipeg, Manitoba \\ [Received for publication May 11, 1961]
}

\begin{abstract}
A total of 865 spring wheat varieties, and selections from the 14, 28 and 42 chromosome groups, were inoculated in 3 successive years with a mixture of Canadian collections of loose smut, Ustilago tritici (Pers.) Rostr. At the end of the first year, 231 were rated immune; after the second inoculation, 188; and after the third, 162 . These included 116 from the 42 chromosome group, 43 from the 28 , and 3 from the 14. Although some of the reduction in immune or resistant types after the first year may have resulted from escapes, it is probable that most of these showed susceptibility to new collections added in the second and third years.
\end{abstract}

\section{INTRODUCTION}

In the early part of this century losses caused by loose smut, Ustilago tritici (Pers.) Rostr., were quite appreciable in North America. Tapke (2) estimates that, in the period 1917-1926, annual losses from this disease were more than 10,000,000 bushels in the United States. No comparable figures are available for Western Canada. In recent years, however, loose smut has been relatively unimportant in wheat production in the Prairie Provinces. The principal varieties grown are Thatcher and Selkirk, which are immune and highly resistant, respectively. The only highly susceptible variety released in the last decade was Lee which, at its widest distribution in 1954, occupied only 20 per cent of the Manitoba and a smaller percentage of the Saskatchewan acreage.

Tapke (2) listed the reaction for 234 varieties and lines available to him in 1929. Of these, he found 13 which were immune. In 1957, observation rows of nearly 1,100 spring wheat varieties were grown in the field at Winnipeg in order to determine their possible value as plant breeding or genetic material. As many of the varieties as possible were inoculated to a mixture of loose smut collections to assess their resistance. In this paper are listed those varieties which remained immune after 3 years of inoculation.

\section{MATERIALS AND METHODS}

The wheat varieties grown in the 1957 genetic nursery were obtained from 28 countries of the world. One or two heads of 865 of the spring types were inoculated with a mixture of 26 collections of loose smut gathered throughout Canada in 1956.

Inoculation was effected by means of a Meyer's Silver Prince sprayer which was set to deliver the spore suspension at a pressure of 270 pounds per square inch (1) through a fine jet nozzle. In this method the wheat head is held in one hand while the spray is directed at the florets. The high pressure of the jet stream forces the glumes open, allowing the inoculum to enter the florets. The operation was carried out 1 or 2 days following anthesis of the central spikelets of selected heads.

${ }^{1}$ Contribution No. 68 of the Canada Department of Agriculture Research Station, Winnipeg, Man. 
The inoculum was prepared by shaking equal numbers of smutted heads of each collection in a pail of water and straining the suspension into the sprayer tank through a screen of sufficiently large mesh to allow spores to pass through but to hold back rachis and floral parts. Enough water for a 4-hour supply was added. Fresh inoculum was prepared after 4 hours' use to ensure viability of the spores.

In 1958 the inoculated seed of each variety was sown in hills in a field nursery and the plants examined for smut infection. If even one head were smutted the variety was discarded. Where no infection occurred, one or two heads were again inoculated, this time with 26 collections of loose smut which had been collected in 1957 . The inoculated seed was sown in 1959 and treated in a similar manner. The procedure was repeated in 1960 making a total of 3 successive years of testing.

RESULTS AND DISCUSSION

Of the 865 inoculated varieties grown in 1958 , only 231 were rated immune. These were further reduced in 1959 to 188 and, after elimination in 1960,162 remained. Of these, 116 were in the 42 chromosome group, 43 in the 28 , and 3 in the 14 . These varieties are grouped in Table 1 according to chromosome number. Where possible, sub-groups such as Thatcher and its derivatives are placed together. Certain agronomic and disease reaction data, recorded in 1957, are included to show the relative value of the varieties for characteristics other than loose smut resistance.

A. B. Campbell* has shown that Thatcher possesses a dominant gene for loose smut immunity. This is reflected in the immunity displayed by Thatcher and its derivatives. Other groups of spring wheat include some of the Kenya varieties and varieties with Supremo, Africa 43 or Maria Escobar as a common parent. None of the varieties listed in Table 1 was tested by Tapke (2).

A narrow genetic base was used for screening each variety. In the first year only one or two heads were inoculated and, since succeeding inoculations were made on plants grown from seed which had been inoculated, all tests were made on plant progenies tracing directly to these original heads. Thus, if the variety were heterogeneous for resistance, and an immune genotype were selected by chance, the variety would be considered immune. Heterogeneity for loose smut resistance may be more widespread than is usually realized. Unless wheat breeding programs are designed to incorporate loose smut resistance, no selection is made for this characteristic, and a variety may be released which carries both resistant and susceptible lines. In many tests for varietal reaction, some varieties are immune, others carry trace to 30 per cent smut, while still others are completely or nearly completely susceptible. The question is whether all those in the trace-to-30 per cent group are susceptible, or whether only a portion of the population is susceptible. Physiologic specialization in Ustilago tritici may provide a second alternative. This organism passes through a sexual cycle in each generation and as a result many races may be present. Thus, in those cases where 80 to 100 per cent infection is observed,

- Personal communication 
TABLE 1. - Agronomic and DISEASE Data For 1957 on VARIETIES AND SELECTIONS OF SPRING WHEAT IMMUNE TO LOOSE SMUT FOR A 3-YEAR PERIOD

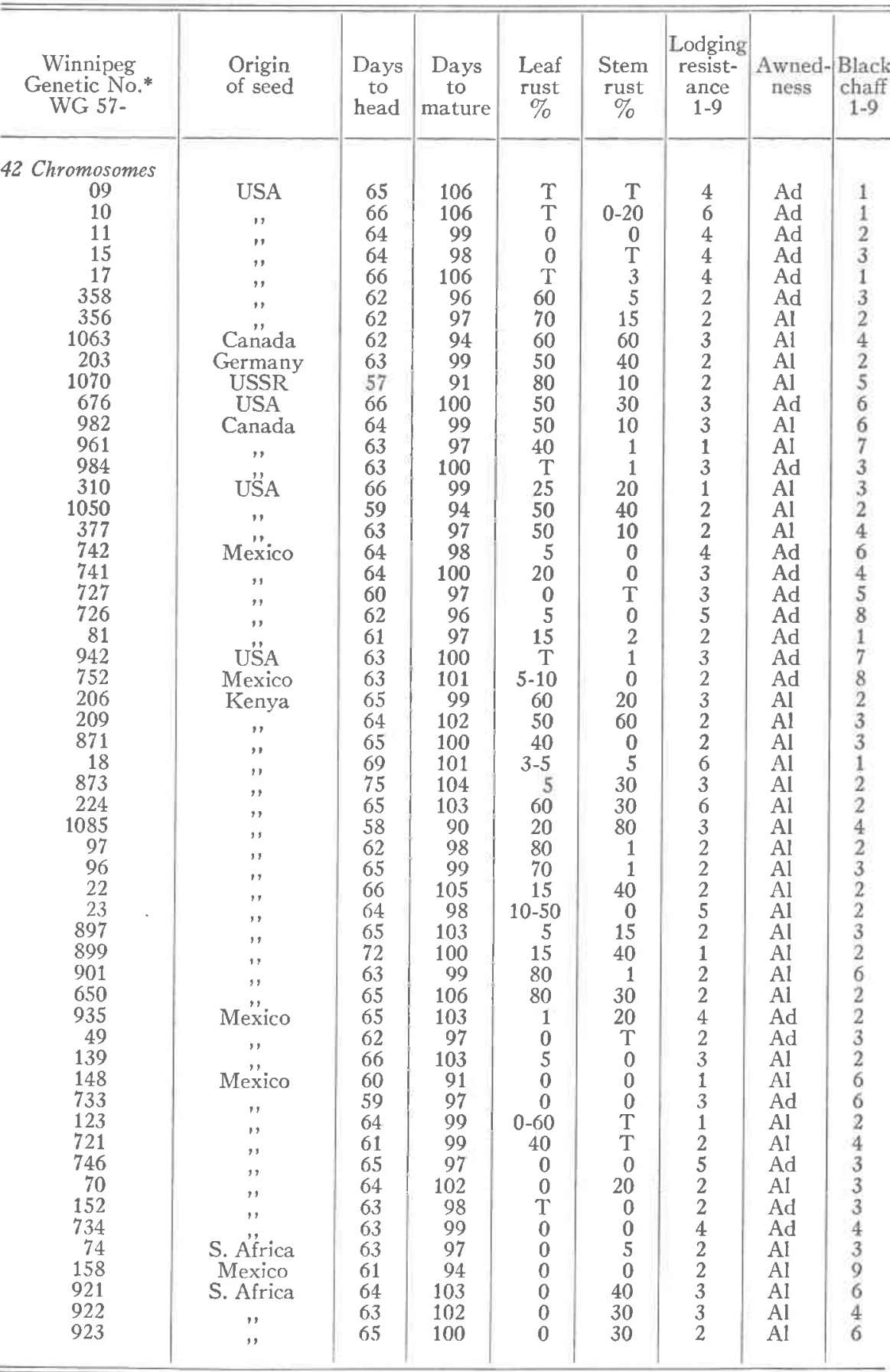


TABlE 1. - continued

\begin{tabular}{|c|c|c|c|c|c|c|c|c|}
\hline $\begin{array}{l}\text { Winnipeg } \\
\text { Genetic No.* } \\
\text { WG 57- }\end{array}$ & $\begin{array}{l}\text { Origin } \\
\text { of seed }\end{array}$ & $\begin{array}{l}\text { Days } \\
\text { to } \\
\text { head }\end{array}$ & $\begin{array}{c}\text { Days } \\
\text { to } \\
\text { mature }\end{array}$ & $\begin{array}{l}\text { Leaf } \\
\text { rust } \\
\%\end{array}$ & $\begin{array}{c}\text { Stem } \\
\text { rust } \\
\%\end{array}$ & $\begin{array}{c}\text { Lodging } \\
\text { resist- } \\
\text { ance } \\
1-9\end{array}$ & $\begin{array}{c}\text { Awned- } \\
\text { ness }\end{array}$ & $\begin{array}{l}\text { Black } \\
\text { chaff } \\
1-9\end{array}$ \\
\hline \multicolumn{9}{|l|}{42 Chromosomes } \\
\hline 924 & S. Africa & 65 & 100 & 0 & 25 & 3 & $\mathrm{Al}$ & 5 \\
\hline 925 & " & 64 & 102 & 0 & 40 & 1 & Al & 4 \\
\hline 926 & ," & 64 & 102 & 0 & 30 & 1 & $\mathrm{Al}$ & 3 \\
\hline 927 & ", & 65 & 103 & 0 & 40 & 1 & Al & 4 \\
\hline 928 & ," & 65 & 103 & 0 & 25 & 2 & $\mathrm{Al}$ & 4 \\
\hline 929 & ," & 65 & 103 & 0 & 10 & 1 & $\mathrm{Al}$ & 2 \\
\hline 930 & ", & 65 & 98 & 0 & 5 & 2 & Al & 3 \\
\hline 931 & ", & 66 & 104 & 0 & 20 & 2 & $\mathrm{Al}$ & 2 \\
\hline 932 & ", & 65 & 103 & 0 & 10 & 5 & $\mathrm{Al}$ & 3 \\
\hline 933 & ," & 62 & 95 & 50 & 1 & 6 & Ad & 7 \\
\hline 107 & S. Africa & 64 & 98 & 0 & 5 & 1 & Ad & 2 \\
\hline 1076 & USA & 59 & 93 & 50 & 5 & 5 & Ad & 5 \\
\hline 939 & Peru & 62 & 99 & 0 & 15 & 3 & Ad & 3 \\
\hline 94 & ", & 63 & 96 & $\mathrm{~T}$ & 0 & 1 & $\mathrm{Ad}$ & 4 \\
\hline 801 & ," & 64 & 99 & $\mathrm{~T}$ & 0 & 2 & Ad & 7 \\
\hline 802 & 3 & 63 & 98 & 1 & 0 & 2 & Ad & 8 \\
\hline 1072 & US̈A & 59 & 94 & 60 & 10 & 4 & Ad & 2 \\
\hline 835 & Colombia & 63 & 97 & $\mathrm{~T}$ & 0 & 4 & $\mathrm{Al}$ & 6 \\
\hline 111 & Argentina & 65 & 101 & 5 & 0 & 4 & Ad & 3 \\
\hline 214 & , & 65 & 98 & 0 & 80 & 3 & Ad & 2 \\
\hline 233 & ". & 65 & 99 & $\mathrm{~T}$ & 70 & 4 & Ad & 2 \\
\hline 704 & $"$ & 63 & 98 & 0 & $\mathrm{~T}$ & 5 & Ad & 8 \\
\hline 245 & USA & 66 & 101 & 40 & 5 & 4 & $\mathrm{Al}$ & 4 \\
\hline 674 & , & 61 & 99 & 30 & 5 & 3 & $\mathrm{Al}$ & 8 \\
\hline 675 & ", & 65 & 97 & 50 & 20 & 4 & $\mathrm{Al}$ & 4 \\
\hline 1005 & Canada & 58 & 92 & 10 & 5 & 3 & Seg. & 5 \\
\hline 682 & USA & 60 & 104 & 5 & 0 & 1 & Ad & 2 \\
\hline 776 & ", & 60 & 103 & 1 & 0 & 2 & Ad & 4 \\
\hline 778 & ", & 61 & 104 & 5 & 0 & 3 & Al & 5 \\
\hline 673 & , & 61 & 107 & 40 & 0 & 7 & Ad & 3 \\
\hline 340 &, & 64 & 99 & 80 & 50 & 5 & $\mathrm{Ad}$ & 4 \\
\hline 347 & ", & 64 & 99 & 70 & $\mathrm{~T}$ & 3 & Ad & 2 \\
\hline 350 & ,, & 63 & 97 & 40 & 90 & 3 & $\mathrm{Ad}$ & 6 \\
\hline 646 & ", & 63 & 99 & 50 & 40 & 2 & $\mathrm{Ad}$ & 2 \\
\hline 545 & Itäly & 62 & 96 & 5 & 90 & 1 & $\mathrm{Ad}$ & 4 \\
\hline 661 & & 64 & 95 & 0 & 50 & 2 & Seg. & 4 \\
\hline 519 & Switzerland & 63 & 95 & $30-90$ & 100 & 2 & $\mathrm{Ad}$ & 2 \\
\hline 522 & , & 64 & 97 & 30 & 80 & 1 & Seg. & 3 \\
\hline 517 & ", & 64 & 98 & 50 & 50 & 1 & Səg. & 4 \\
\hline 518 & ," & 64 & 98 & 30 & 40 & 1 & Seg. & 2 \\
\hline 630 & Canada & 61 & 97 & 70 & 40 & 2 & $\mathrm{Ad}$ & 2 \\
\hline 821 & Chile & 65 & 102 & 30 & 0 & 2 & Al & 6 \\
\hline 823 & ,. & 61 & 99 & 1 & 0 & 3 & $\mathrm{Al}$ & 8 \\
\hline 824 & ,", & 62 & 107 & 1 & 0 & 2 & Ad & 3 \\
\hline 825 & ", & 61 & 106 & $\hat{\mathrm{T}}$ & 0 & 2 & $\mathrm{Ad}$ & 6 \\
\hline 822 & 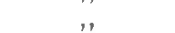 & 61 & 99 & 10 & 0 & 3 & $\mathrm{Al}$ & 3 \\
\hline 830 & Colombia & 71 & 107 & 5 & 25 & 2 & $\mathrm{Al}$ & 4 \\
\hline 831 &  & 61 & 95 & 3 & 5 & 3 & Al & 7 \\
\hline 832 & & 64 & 98 & 60 & $\mathrm{~T}$ & 5 & Al & 8 \\
\hline 1024 & Australia & 60 & 88 & 100 & 100 & 2 & Al & 5 \\
\hline 183 & Here & 61 & 92 & 90 & 100 & 2 & Al & 3 \\
\hline 443 & Canada & 62 & 93 & 60 & 90 & 1 & AI & 2 \\
\hline 422 & & 68 & 96 & 80 & 90 & 2 & $\mathrm{Al}$ & 2 \\
\hline 625 & Eire & 68 & 99 & 40 & 90 & 1 & Al & 2 \\
\hline 87 & Canada & 60 & 97 & $10-80$ & 70 & 1 & $\mathrm{Al}$ & 2 \\
\hline 1054 & USA & 59 & 88 & 10 & 100 & 3 & $\mathrm{Ad}$ & 2 \\
\hline
\end{tabular}


TABLE 1. - continued

\begin{tabular}{|c|c|c|c|c|c|c|c|c|}
\hline $\begin{array}{l}\text { Winnipeg } \\
\text { Genetic No.* } \\
\text { WG 57- }\end{array}$ & $\begin{array}{l}\text { Origin } \\
\text { of seed }\end{array}$ & $\begin{array}{l}\text { Days } \\
\text { to } \\
\text { head }\end{array}$ & $\begin{array}{c}\text { Days } \\
\text { to } \\
\text { mature }\end{array}$ & $\begin{array}{l}\text { Leaf } \\
\text { rust } \\
\%\end{array}$ & $\begin{array}{l}\text { Stem } \\
\text { rust } \\
\%\end{array}$ & $\begin{array}{c}\text { Lodging } \\
\text { resist- } \\
\text { ance } \\
1-9\end{array}$ & $\begin{array}{l}\text { Awned- } \\
\text { ness }\end{array}$ & $\begin{array}{l}\text { Black } \\
\text { chaff } \\
1-9\end{array}$ \\
\hline $\begin{array}{c}42 \text { Chromosomes } \\
758 \\
1143 \\
498 \\
1110 \\
490\end{array}$ & $\begin{array}{c}\text { Mexico } \\
\text { Czechoslovakia } \\
\text { Canada } \\
\text { Czechoslovakia } \\
\text { Canada }\end{array}$ & $\begin{array}{l}60 \\
55 \\
67 \\
62 \\
70\end{array}$ & $\begin{array}{r}97 \\
84 \\
97 \\
94 \\
110\end{array}$ & $\begin{array}{r}15 \\
100 \\
30 \\
30 \\
15\end{array}$ & $\begin{array}{r}0 \\
100 \\
100 \\
100 \\
90\end{array}$ & $\begin{array}{l}3 \\
3 \\
3 \\
3 \\
3\end{array}$ & $\begin{array}{l}\mathrm{Al} \\
\mathrm{Ad} \\
\mathrm{Ad} \\
\mathrm{Ad} \\
\mathrm{Ad}\end{array}$ & $\begin{array}{l}8 \\
8 \\
2 \\
3 \\
2\end{array}$ \\
\hline $\begin{array}{c}28 \text { Chromosomes } \\
343 \\
344 \\
387 \\
391 \\
683 \\
685 \\
690 \\
810 \\
811 \\
812 \\
816 \\
808 \\
807 \\
688 \\
817 \\
839 \\
944 \\
691 \\
945 \\
947 \\
951 \\
949 \\
950 \\
952 \\
1152 \\
703 \\
304 \\
312 \\
482 \\
483 \\
485 \\
1121 \\
1125 \\
1128 \\
1126 \\
486 \\
1017 \\
837 \\
1049 \\
1051 \\
445 \\
1095 \\
1096 \\
\end{array}$ &  & $\begin{array}{l}64 \\
64 \\
62 \\
62 \\
64 \\
67 \\
63 \\
63 \\
62 \\
64 \\
64 \\
61 \\
68 \\
68 \\
65 \\
68 \\
62 \\
62 \\
62 \\
63 \\
65 \\
65 \\
66 \\
63 \\
60 \\
62 \\
60 \\
73 \\
70 \\
69 \\
69 \\
66 \\
66 \\
68 \\
62 \\
66 \\
62 \\
68 \\
60 \\
59 \\
75 \\
62 \\
61\end{array}$ & $\begin{array}{r}97 \\
95 \\
97 \\
97 \\
100 \\
106 \\
101 \\
94 \\
95 \\
95 \\
99 \\
95 \\
95 \\
107 \\
99 \\
106 \\
90 \\
99 \\
94 \\
95 \\
96 \\
95 \\
97 \\
94 \\
95 \\
95 \\
94 \\
102 \\
100 \\
100 \\
100 \\
100 \\
95 \\
102 \\
94 \\
95 \\
92 \\
102 \\
93 \\
93 \\
96 \\
93 \\
95\end{array}$ & $\begin{array}{r}0 \\
0 \\
0 \\
0 \\
0 \\
0 \\
0 \\
0 \\
0 \\
0 \\
0 \\
0 \\
0 \\
0 \\
0 \\
0 \\
5 \\
\mathrm{~T} \\
1 \\
\mathrm{~T} \\
5 \\
0 \\
0 \\
0 \\
\mathrm{~T}-5 \\
0 \\
\mathrm{~T} \\
5 \\
\mathrm{~T} \\
2 \\
0 \\
5 \\
\mathrm{~T} \\
0 \\
0 \\
40 \\
80 \\
0 \\
0 \\
2 \\
5 \\
0 \\
0\end{array}$ & $\begin{array}{r}30 \\
\mathrm{~T} \\
\mathrm{~T} \\
15 \\
0 \\
5 \\
1 \\
0 \\
15 \\
0 \\
0 \\
0 \\
2 \\
30 \\
0 \\
40 \\
0 \\
0 \\
0 \\
0 \\
\mathrm{~T} \\
0 \\
\mathrm{~T} \\
0 \\
-\mathrm{T} \\
0 \\
10 \\
40 \\
90 \\
60 \\
100 \\
80 \\
80 \\
90 \\
100 \\
50 \\
0 \\
40 \\
40 \\
90 \\
20 \\
70\end{array}$ & $\begin{array}{l}4 \\
4 \\
5 \\
5 \\
4 \\
8 \\
9 \\
7 \\
5 \\
7 \\
7 \\
6 \\
8 \\
\end{array}$ & $\begin{array}{l}\text { Ad } \\
\text { Ad } \\
\text { Ad } \\
\text { Ad } \\
\text { Ad } \\
\text { Ad } \\
\text { Ad } \\
\text { Ad } \\
\text { Ad } \\
\text { Ad } \\
\text { Ad } \\
\text { Ad } \\
\text { Ad } \\
\text { Ad } \\
\text { Ad } \\
\text { Ad } \\
\text { Ad } \\
\text { Ad } \\
\text { Ad } \\
\text { Ad } \\
\text { Ad } \\
\text { Ad } \\
\text { Ad } \\
\text { Ad } \\
\text { Ad } \\
\text { Ad } \\
\text { Ad } \\
\text { Ad } \\
\text { Ad } \\
\text { Ad } \\
\text { Ad } \\
\text { Ad } \\
\text { Ad } \\
\text { Ad } \\
\text { Ad } \\
\text { Ad } \\
\text { Ad } \\
\text { Ad } \\
\text { Ad } \\
\text { Ad } \\
\text { Ad } \\
\text { Ad } \\
\text { Ad }\end{array}$ & $\begin{array}{l}3 \\
3 \\
3 \\
3 \\
2 \\
2 \\
5 \\
3 \\
3 \\
3 \\
4 \\
5 \\
5 \\
3 \\
5 \\
5 \\
3 \\
5 \\
5 \\
5 \\
2 \\
7 \\
4 \\
4 \\
3 \\
6 \\
6 \\
4 \\
4 \\
4 \\
5 \\
4 \\
4 \\
5\end{array}$ \\
\hline $\begin{array}{c}14 \text { Chromosomes } \\
1073 \\
447 \\
694\end{array}$ & $\begin{array}{c}\text { USA } \\
\text { Canada } \\
\text { USA }\end{array}$ & $\begin{array}{l}59 \\
69 \\
65\end{array}$ & $\begin{array}{r}94 \\
107 \\
100\end{array}$ & $\begin{array}{l}0 \\
0 \\
\mathrm{~T}\end{array}$ & $\begin{array}{r}0 \\
1 \\
60\end{array}$ & $\begin{array}{l}6 \\
4 \\
8\end{array}$ & $\begin{array}{l}\text { Ad } \\
\text { Ad } \\
\text { Ad }\end{array}$ & $\begin{array}{l}1 \\
1 \\
2\end{array}$ \\
\hline
\end{tabular}

*Variety or selection names 
42 Chromosomes

09 Frontana-Thatcher

10

11

15

17

,

"

"

$358 \mathrm{H}-44 \times$ Thatcher

356 Thatcher

1063 Thatcher selection

203 Hochzucht (similar to Thatcher)

1070 Kostoff's triple hybrid (sim. to That.)

676 Pilot $^{2} \mathrm{x}$ 'Thatcher

982 Thatcher x Regent

961 Thatcher $^{6}$ x Kenya Farmer

927 Red Egyptian Type 170918

928

929

930

931

932

933

107

1076 H-44-24

939 Maria Escobar

94 Maria Escobar x H-44-Marquis

801

802

984 (Canus $\mathrm{x}$ Thatcher) $\mathrm{x}$ (482.B x Regent)

310 Double Cross 825-4

1050 Marquillo x Waratah

377 New thatch

742 (Mentana x Merit-Kenya) x (Kenya-Gular-Pilot) (Kenya 58-Newthatch)

741 Merit (Newthatch-Marroqui) Kentana $x$ (Kenya-Gular-Pilot) $\mathbf{x}$ (Kenya 58-Newthatch)

727 (Kenya-Redman-Dundee) $x$ Newthatch

726 Candeal-Newthatch-Kentana

81 (Kenya-Gular-Pilot) $x$

942 Willet (Kenya 58-Newthatch)

752 Willet $x$ Lerma

206 Kenya I-40-34

209 ," I-40-45

$871 \quad$ " I-49-157

18 " $117 \mathrm{~A}$

873 " 117A I-49-161

224 " 235.A.1.A.4

1085 " B.256.G

B. 286

$\begin{array}{lll}96 & \text { " } \\ 22 & , & 321 \cdot \text { B. } 2 . A .3 \\ 23 & , 1 . C .3\end{array}$

23 " 321. BT.1.B.1

897 "338.Z.2.G.2

899 340.Y.4.A.1

901 340.Y.6.B.3

650 " $"$ 351.AS.1.B.2

935 Supremo

49 Peru x Supremo

139 Supremo-Kenya x Egypt-Timstein

148 Supremo-Timstein-Kenya

733 Supremo $211 \times$ Lerma

123 Supremo $^{2}$ x Kenya

721 Kenya-Supremo x Yaqui

746 Wisc. $245 \times$ Supremo 51

\section{Chromosomes}

70 (Mayo x Peru-Supremo) $\mathrm{x}$ (Peru-Kenya)

152 Mayo (Pelon, Colorado-Renown ${ }^{2}-$ Supremo) Kentana ${ }^{2}$

734

74 Africa 43

158 Africa $43 \times$ Yaqui 50

921 Red Egyptian Type 170915

922 ", 170915-3c

$923 \quad$ ", 170916

924 170916-2c

$925 \quad$ " 170917

$926 \quad 170917-2 \mathrm{c}$

1072 (Ceres-Hope) x Florence

835 Riccio $x$ Yaqui-Kentana

111 Argentina No. 49-4824

214 Klein 40

233 Sinvalocho M.A.

704 Magnif disro

245 Mida-Cadet

674 Lee x 1831

675 Rescue x 1831

1005 (Mida-Cadet) x (McMurachyExchange x Redman), R.L. 2667

682 K 338 AA x Ns 3880-191, ND 52

776 ND 57

$778 \quad$ ND 59

673 Lee-Frontana II-47-10

340 Rival

347 Mercury

350 Premier

646 Russell

545 Oleata Calandria

661 Leone

519 B 1

522 B 15

517 263-25-2

$518 \quad 263-27-2$

630 C.D. $3872-4$. C.A.N. 3740

821 Lleafen

823 Rosella

$82476 \mathrm{R}$

$82522 \mathrm{R}$

822 Redondo Negro

830 Frontana-Egypt $\mathrm{Na} 95$

831 (Mida-Kenya 117 A) Frontana

832 Salles-McMurachy x Mayo 48

1024 Bunyip

183 Bobs

443 Red Bobs

422 Renfrew

625 Rosa Karn x Karn II

87 Cailloux

1054 Chinese $x$ Webster

758 (Timstein-Kenya $58 \times$ Gabo) Lee

$1143 T$. vulgare Vill.

$498 T$. aestivum L. var. ferrugineum

1110 T. compactum Host.

$490 T$. spelia L. var. coeruleum

\section{Chromosomes}

343 Gaza

344

387 Patrizio 6

391 Capeiti 8

683 Yuma, Ld. 364

685 Towner, Ld. 370 
28 Chromsomes

690 Sentry

810 C.I. $3255 \times$ Khapli-D.C. 50

811

812

816 Khapli-D.C. "x Carleton 50

808 Ld. 340-Khapli x P.I. 94701

807 Golden Ball-Iumillo-Mindum, R.L. 1714

688 R.L. 3206

817 Medeah AP 9

839 Tremes preto

944 Amarai bianco tipo

691 St. 464

945 C.I. 7805

947 C.I. 7875

951 Camadi

949 Rojal de Almeria

950 Tounse

952 Arabian

1152 Giza

703 Beladi 116

304 Khapli

312 Black Persian

$482 T$. durum var. affine

483 T. durum var, hordeiforme

$485 T$. durum var. leucomelan

1121 T. turgidum L. 0-10-868

1125 T. turgidum L. 0-10-769

1128 T. turgidum L. 0-10-751

1126 T. turgidum L. 0-10-824

$486 T$. turgidum L. var. gentile

$1017 T$. dicoccoides I-38-55

837 Yaroslav emmer

1049 Emmer R.L. 116

1051 Early emmer R.L. 114

$445 T$. polonicum var. levissimum

$1095 T$. pyramidale 0-10-911

$1096 T$. pyramidale 0-10-920

14 Chromosomes

$1073 T$. aegilopoides I-38-21

$447 T$. monococcum var. hornemanii 694 Einkorn C.I. 2433 
the variety may be susceptible to many biotypes, but where a low level is observed the variety may be susceptible to only a few of these: only those seeds infected by a virulent biotype give rise to smutted plants. New biotypes, therefore, may explain the sudden susceptibility of a number of immune varieties observed in the present investigation, since some new collections were included in the mixture each year.

Inoculation by means of a high pressure jet sprayer can be considered quite effective as many of the more susceptible varieties carried up to 100 per cent infection in the first year of testing. However, more refined tests using needle inoculation might possibly reveal some susceptibility in the varieties which were retained. Such a procedure should be followed before the varieties reported herein as immune are used in a program breeding for resistance or in genetic studies for inheritance of resistance. Subject to these qualifications, it is considered that these varieties will be of value in the plant breeding and genetic fields.

\section{ACKNOWLEDGEMENTS}

The author is indebted to W. J. Cherewick for supplying inoculum of the different collections of loose smut, and to J. R. Murray who assisted in the inoculations.

\section{REFERENCES}

1. Moore, M. B., and D. E. Munnecke. High pressure jet inoculation of wheat and barley with loose smut. Minn. Agr. Expt. Sta. Misc. J. Series 665.

2. Tapke, V.F. Influence of varietal resistance, sap acidity and certain environmental factors on the occurrence of loose smut in wheat. J. Agr. Research 39:313-339. 1929. 